\title{
The role of balanced supplementation in diet of age-related macular degeneration patients
}

\author{
Iwona Kusz vel Sobczuk, Anna Święch
}

Medical University of Lublin, Department of Retinal and Vitreous Surgery Head: Prof. Jerzy Mackiewicz, MD, PhD

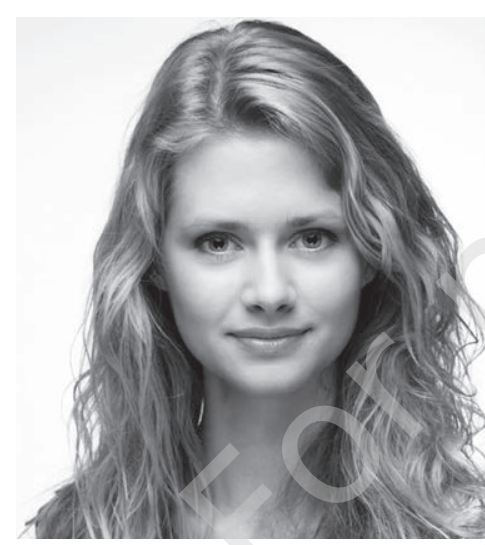

\section{ABSTRACT}

Aim: The aim of the article was to discuss the role of balanced supplementation in diet of age-related macular degeneration patients.

Methods: This review was carried out using comprehensive and systematic literature reports on the role of supplementation of vitamin D, vitamin C, vitamin $E$, vitamin $B_{6}$, vitamin $B_{12}$, zinc, lutein, zeaxanthin, omega-3 acid and folic acid in the prevention of AMD.

Results: Vitamins, minerals and carotenoids are essential for the proper retinal function over an inflammation and immune response modulation.

Conclusions: Vitamins, minerals and carotenoids discussed in the article have anti-inflammatory and antioxidative properties in the management of AMD progression. Accordingly, it is relevant to assure the appropriate level of these nutrients in a diet of AMD patients.

Key words: AMD, vitamins, minerals, carotenoids 


\section{INTRODUCTION}

Age-related macular degeneration (AMD) is one of the main causes of vision loss in developed countries, including Poland. This disease affects mainly people aged over 50, and its morbidity rate increases with age. AMD is classified into two types: dry and wet (exudative). The dry form accounts for about $85-90 \%$ of all AMD cases, whereas the wet form - for about $10-15 \%$. This progressive disease is characterised by degeneration of the retinal pigment epithelium (RPE), leading to photoreceptor death and central vision loss. Retinal pigment epithelium cells are prone to oxidative stress - a factor that induces inflammation. The dry form is characterised by the presence of drusen, i.e. metabolic products that accumulate under the retinal pigment epithelium, and a thickening of the Bruch's membrane. Dry AMD also entails geographic atrophy with discrete regions of loss of RPE, photoreceptors, and choroidal capillaries. Wet AMD is characterized by the presence of choroidal neovascularization - CNV. The formation of the subretinal neovascular membrane occurs through the pathway of pathological angiogenesis. The vascular endothelial growth factor (VEGF) plays a major role among the factors activating this process [1].

The gold standard in the treatment of wet AMD are intravitreal injections of anti-VEGF inhibitors. This treatment is intended to inhibit the formation of new vessels in the subretinal space. Unfortunately, there is currently no effective therapy for dry AMD.

The etiology of AMD has not yet been fully understood. Based on the available epidemiological studies, it has been found to be multifactorial. It is believed that apart from the aging processes, genetic predisposition and certain environmental factors, such as smoking, diet, ultraviolet radiation or concomitant cardiovascular diseases, are also important [2].

An appropriate, varied and age-suitable diet is fundamental to leading a healthy lifestyle. Dietary intake of lutein, zeaxanthin, omega-3 acids, folic acid, zinc and vitamins $\mathrm{D}, \mathrm{B}_{6}, \mathrm{~B}_{12}, \mathrm{C}$ and $\mathrm{E}$ is known to have a beneficial effect on maintaining normal vision. The aforementioned vitamins, minerals and carotenoids have anti-inflammatory, anti-oxidative and immunomodulating properties, and reduce the risk of AMD progression. As the human body is ageing, the uptake and assimilation of nutrients decreases. This article discusses the relation between the supply of particular nutrients and the risk of AMD progression.

\section{VITAMIN D}

Vitamin D precursor is produced mainly in the skin (approx. $80-100 \%$ ). The vitamin is formed under ultravio- let light (UV) as a result of its precursor 7-dehydrocholesterol (7DHC) being converted into vitamin $\mathrm{D}_{3}$. The amount of human-made vitamin $\mathrm{D}$ is subject to seasonal fluctuations. In the moderate climate zone, the amount of sunlight supplied for about half of the year is too little for human skin to produce enough of this vitamin. An alternative source of vitamin D is diet, covering about $20 \%$ of the daily demand. Its two forms are found in food products: vitamin D of animal origin - cholecalciferol (vitamin $\mathrm{D}_{3}$ ) and vitamin $\mathrm{D}$ of plant origin - ergocalciferol (vitamin $\mathrm{D}_{2}$ ). Cholecalciferol, which is absorbed in the small intestine, is transported to the liver and binds to DBP (vitamin D binding protein). The liver is responsible for the first stage of the biosynthesis of the active form of vitamin D. After enzymatic hydroxylation, vitamin $25-(\mathrm{OH}) \mathrm{D}$ is formed. This reaction is catalyzed by 25-hydroxylase (CYP27A1, CYP3A4, and CYP2R1), which is a group of hydroxylases of cytochrome $\mathrm{P} 450$. Vitamin 25- $(\mathrm{OH}) \mathrm{D}$ is transported to the kidneys, where an active form of vitamin $\mathrm{D}-1_{\alpha} 25(\mathrm{OH}) 2 \mathrm{D}$ (calcitriol) is formed, in the presence of $1_{\alpha}$-hydroxylase (CYP27B1). Calcitriol belongs to a broad group of hormones which are transcription factors for genes of target proteins. Calcitriol exerts its biological action through the intracellular receptor VDR (vitamin D receptor). The discovery of vitamin D receptor's presence in many tissues initiated research on other non-classical and extraskeletal effects of vitamin $\mathrm{D}$.

In 2014, a paper was published documenting the presence of the VDR receptor in human eye tissues and its potential role in the synthesis of vitamin $\mathrm{D}$ [3]. This team assessed human scleral fibroblasts (HSF), human corneal endothelial cells-12 (HCEC-12), nonpigmented ciliary body epithelial (ODM-2) cells and adult retinal pigment epithelial-19 (ARPE-19) cells for the expression of, among other things, the vitamin D receptor (VDR), enzymes activating vitamin $\mathrm{D}_{3}\left(1_{\alpha}\right.$-hydroxylase-CYP27B1, 25-hydroxylase-CYP27A1 and CYP2R1), using RT-PCR (real-time polymerase chain reaction), immunocytochemistry and immunoenzyme assays. The study showed that all the aforementioned cell types demonstrate mRNA and protein expression for all components synthesizing and metabolising vitamin D. All cell types except human scleral fibroblasts have the ability to convert inactive 25-hydroxyvitamin $\mathrm{D}_{3}$ into its active form in vitro [3]. Vitamin $\mathrm{D}_{3}$ has been classified as an immunomodulatory hormone. It controls innate and acquired immune responses [4]. Calcitriol inhibits T CD4+ cell production of proinflammatory cytokines in vitro, and promotes regulatory $\mathrm{T}$ cell phenotype [5]. Endogenous conversion of $25(\mathrm{OH}) \mathrm{D}_{3}$ has been shown to inhibit dendritic cell antigen presentation and chemotaxis [6]. 
Vitamin D is an immune system modulator and cooperates with two members of family $\mathrm{H}$ and I regulators of complement activation [7]. Calcitriol is believed to modulate the adaptive immune response to inhibit inflammation. Additionally, it reduces proinflammatory cytokine production in immune cells, inhibits dendritic cell maturation, as well as $\mathrm{T}$ and $\mathrm{B}$ cells proliferation. In 2015, Amy E. Millen and her team conducted the study CAREDS, describing the synergistic effect between the level of vitamin $\mathrm{D}$ and the expression of the complement cascade proteins. These researchers further demonstrated that the polymorphism of proteins which are necessary to activate the complement cascade increases the risk of AMD. An approximately 6-fold increase has been observed in the probability of AMD in women with vitamin D deficiency (<30 nmol/l) and 2 risk alleles for complement factors $\mathrm{H}$ and $\mathrm{I}$, as compared to women who do not carry the high risk alleles with an appropriate level of vitamin D (> $75 \mathrm{nmol} / \mathrm{l})$ [8].

The 2018 recommendations of the Polish Society of Pediatric Endocrinology and Diabetes and of the expert panel with the participation of national expert consultants and representatives of scientific societies recommend individual vitamin $\mathrm{D}$ dosage titration, according to the age, body weight, sunlight (time of year), sunlight exposure, eating habits and lifestyle. In healthy adults (19-65 years old) who sunbathe with exposed forearms and legs for at least 15 minutes between 10 am and 3 pm, with no sunblock, from May to September, supplementation is not necessary, although it is still recommended and safe. If the above recommendations are not followed, doses of 800-2000 IU/day based on body weight and dietary consumption of vitamin $\mathrm{D}$ throughout the year are recommended. Due to reduced effectiveness of dermal synthesis in the elderly (> 65-75 years old) and people with dark complexion, vitamin D supplementation is recommended at the dose of 800-2000 IU/24 h, depending on body weight and dietary intake of the vitamin throughout the year [9]. In the oldest people (> 75 years old), due to reduced efficacy of dermal synthesis, potential absorption disorders and altered vitamin D metabolism, it is recommended to supplement it with a dose of 2000-4000 IU/24 h, depending on body weight and diet, throughout the year. In Poland, skin synthesis of vitamin D can only be effective in spring and summer (from May to September). In a large part of the adult population exposure to sunlight is typically very limited due to the type of work performed [9].

Vitamin D has immunomodulating and anti-inflammatory properties, and thus reduces the risk of AMD. Supplementation of vitamin D in the adult population is recommended due to the limited potential for its dermal synthesis. This results from a limited exposure to sun- light due to the type of work performed and the latitude of Poland.

\section{CAROTENOIDS - LUTEIN AND ZEAXANTHIN}

In the human body, there is no lutein synthesis de novo, it is supplied with food only. Carotenoids are fat soluble and therefore high fat diet facilitates their absorption. Once these compounds are supplied and dissolved in the gastric juice, they are absorbed in the form of micelles. The transport takes place mainly through low-density lipoproteins (LDL 55\%), high density lipoproteins (HDL 33\%) and very low density lipoproteins (VLDL 10-19\%) [10]. The distribution of lutein and zeaxanthin in LDLs and HDLs is similar. In a cross-sectional study published in 2012, a relation was observed between lutein or zeaxanthin concentrations and lipoproteins. Moreover, it was found that a change in lipoprotein concentrations may affect lutein and zeaxanthin levels in the retina [11]. The transport of lutein to various human tissues is not uniform. In the human body, lutein and zeaxanthin are particularly concentrated in the macula, where they are main components of the macular pigment. The latest studies carried out using confocal microscopy have shown the spatial distribution of lutein and zeaxanthin in the human retina. Zeaxanthin has been shown to be concentrated in the fovea, extending from the inner to the outer border membrane, with particularly high concentration in the outer plexus layer. The concentration of zeaxanthin decreases sharply in the peripheral part of the macula. Lutein, on the other hand, is more evenly distributed in the macula, at a relatively lower concentration as compared to zeaxanthin [12].

Due to the presence of hydroxyl groups in the carbon ring in its molecular structure, lutein demonstrates higher polarity than other carotenoids. This structure enables it to bond better to the serum oxygen [13]. Lutein and zeaxanthin are a type of blue light high-energy filter, protecting the macula against photooxidative damage. Fluorescence emission has been shown to be lower in carotenoid-containing liposomes than in carotenoids-free controls after exposure to blue light, indicating the filter effect. The filter efficiency was the highest for lutein, followed by zeaxanthin, and to a lesser extent - for $\beta$-carotene [14]. Diet enrichment with additional carotenoids supplementation may reduce the impact of oxidative stress on the retina, reducing the blue light impact intensity by up to $90 \%$. Under physiological conditions, reduction of the harmful effects of blue light on the retina is about 40\% [15]. Nowadays, it is of great importance because our eyes are particularly exposed to toxic blue light from smartphones, computers and LED lamps, which are increasingly popular indoors. 
Numerous studies confirm the anti-inflammatory properties of lutein and zeaxanthin. In vitro studies show that lutein inhibits activation of proinflammatory molecules such as: NF-KB (nuclear factor kappa-light-chain-enhancer of activated B cells), as well as iNOS expression (inducible nitric oxide synthase) and COX-2 (cyclooxigenase-2) [16] and possibly exerts effects on the level of factor $\mathrm{D}$ of the complement activation pathway, which is involved in the pathogenesis of AMD [17]. In addition, lutein and zeaxanthin inhibit the expression of genes related to inflammation [18]. There is evidence of a possible beneficial role of lutein in increasing survival of glial cells after damage caused by hypoxia, through regulating both apoptosis and autophagy [19].

An article published by Gale et al. discusses the relation between lutein and zeoxanthin plasma concentrations and age-related macular degeneration. The increased risk of early and late forms of AMD was significantly higher for people with lower plasma concentrations of zeaxanthin as compared to people without this condition. Low plasma concentrations of lutein and zeaxanthin or lutein alone were associated with a slight increase of the risk of AMD. The study cited above confirms that zeaxanthin can protect against age-related macular degeneration [20].

Lutein has a relatively high safety profile (GRAS, generally recognized as safe), as shown by numerous studies [11]. Based on the AREDS2 study, lutein and zeaxanthin should be taken together, at a proportion of $5: 1$. The recommended dose of lutein is $10 \mathrm{mg}$, and zeaxanthin - $2 \mathrm{mg}$ [21].

Lutein and zeaxanthin will reduce the risk of early and late forms of AMD. They have anti-inflammatory and anti-oxidative properties. Lutein and zeaxanthine are the main component of the macular pigment, constituting a type of blue light high-energy filter and hence protecting the macula against photooxidative damage.

\section{OMEGA-3 ACIDS}

Omega- 3 acids are unsaturated fatty acids. They include e.g. eicosapentaenoic acid (EPA) and docosahexaenoic acid (DHA). The human body is unable to produce them itself. Their source is food, mainly sea fish. Omega-3 and omega-6 fatty acids play a key role in normal brain function and cell growth and differentiation. Omega- 3 acids are components of the cellular and mitochondrial membrane phospholipids, microsomes, nervous system cells and retina. In many studies they have been shown to have anti-inflammatory, cardioprotective, hypotensive, and anti-atherosclerotic effects. The beneficial properties of docosahexaenoic acid and eicosapentaenoic acid suggest a probable mechanism of inflammatory and immune response regulation in the retina. There are many studies showing the beneficial effect of omega-3 acids in reducing the risk of AMD. A study on elderly male twins has been conducted in the United States. It proved that frequent consumption of fish and higher consumption of omega-3 fatty acids reduced the risk of $A M D$, even after considering other risk factors. Increased fish consumption with 2 or more portions per week reduced the risk of AMD. The protective effect of omega-3 long chain polyunsaturated fatty acid absorption was only visible when the consumption of linoleic acid (omega- 6 fatty acid) was low [22]. Similar conclusions can be found in other publications, addressing the need to maintain the appropriate ratio of omega- 6 and omega- 3 fatty acids in the diet. The ideal omega-6/omega-3 ratio is from $3: 1$ to $4: 1$ $[22,23]$. The results presented by Seddon et al. show that an excessive amount of omega- 6 fatty acids in the diet weakens the protective effect of omega- 3 fatty acids. In addition, the negative influence of smoking as a risk factor for AMD was confirmed. Current smokers had an almost 2 times greater risk of AMD, and past smokers had a 1.7 times greater risk than those who had never smoked [22].

Omega- 3 acids reduce the risk of AMD by controlling the inflammatory and immune response in the retina. The protective effect of omega- 3 fatty acids occurs only when an appropriate omega- 6 to omega- 3 acids ratio in the diet is maintained.

\section{FOLIC ACID, $B_{6^{\prime}} B_{12}$}

Homocysteine is an amino acid synthesized from methionine in the body and it is an intermediate product in the cysteine synthesis process. Homocysteine conversions are closely dependent on vitamin $B_{6}, B_{12}$ and folic acid metabolism. The measurement of homocysteine (HCY) concentration is used in risk assessments of atherosclerotic and thrombotic diseases. Increased homocysteine blood level results in endothelial dysfunctions, impairs vascular reactivity, and intensifies inflammatory processes, among other things [24, 25].

Vitamin $B_{12}, B_{6}$ and folic acid deficiencies lead to an increase in $\mathrm{HCY}$ concentration. A potential relation between increased concentrations of serum homocysteine and increased risk of AMD was demonstrated. There is also evidence of a relation between total consumption of vitamin $B_{12}$ and folic acid equivalent and 10-year incidence of AMD. The 10-year increased risk of AMD was significantly associated with baseline vitamin $B_{12}$ or folic acid deficiency. In addition, vitamin $B_{12}$ supplementation was associated with a reduced risk of AMD. These correlations were independent from potential additional factors that could have affected the study, such as smoking, consumption of fish and other dietary supplements, as well as kidney function parameters and white blood cell counts [26].

A large randomised WAFACS study was published in 2009, where Christen et al. assessed the impact of group B vita- 
mins and folic acid intake on the prevalence of AMD. The follow up period was about 7 years. The study group included over 5400 women with an increased risk of cardiovascular diseases. The participants were randomly assigned to one of two groups - folic acid $(2.5 \mathrm{mg} / 24 \mathrm{~h})$, vitamin $\mathrm{B}_{6}$ (50 mg/24 h), and vitamin $B_{12}(1 \mathrm{mg} / 24 \mathrm{~h})$ group or placebo group. The group of women receiving folic acid, vitamin $B_{6}$ and vitamin $B_{12}$ demonstrated a $35-40 \%$ lower AMD risk, as compared to the placebo group. Plasma homocysteine levels were reduced by $18.5 \%$ in the folic acid and vitamins B group, as compared to the placebo group. The beneficial effect of supplementation with folic acid, vitamin $\mathrm{B}_{6}$ and vitamin $B_{12}$ began to appear after approx. 2 years of follow-up, and persisted throughout the remaining period [27]. Folic acid, vitamin $B_{6}$ and vitamin $B_{12}$ supplementation may reduce the risk of AMD by lowering plasma homocystein levels and reducing inflammatory processes. In the elderly, due to poor absorption, the optimal level of vitamin $B_{12}$ may be challenging to achieve. For these patients, the alternative is vitamin $B_{12}$ supplementation, which, if taken at higher doses, is more bioavailable than the naturally occurring vitamin [28].

\section{ANTIOXIDANTS (ZINC, VITAMINS C AND E)}

Zinc is an essential mineral for the human body, as it acts as a catalyst for more than 300 enzymes. It plays an important role e.g. in the functioning of the immune system and coagulation, and it is an important component of the body's antioxidant defense. Zinc has been shown to participate in inhibition of complement activation and increases the anti-oxidative capability of the retina, which may play a protective role in AMD [29]. A study carried out in Australia and published in 2019 by Harshil Dharamdasani Detaram and team confirmed that decreased dietary zinc intake was associated with a higher probability of subretinal fluid presence [30].

Zinc has anti-oxidative and anti-inflammatory properties, which can play a role in the prevention of AMD.

Vitamins $C$ and $E$ are mainly known for their anti-oxidative properties, consisting in eliminating free radicals responsible for DNA damage.

\section{AREDS AND AREDS2 FORMULAS}

AREDS and AREDS2 are the largest multi-centre randomized clinical studies on the role of dietary supplements in AMD patients, carried out on groups of over 4000 people.

AREDS study (Age-Related Eye Disease Study) was designed to assess the impact of antioxidants and/or zinc present in diet on the risk of developing advanced AMD. The participants were randomly assigned to four groups receiving suitable oral preparations daily: group 1 - anti- oxidants (500 mg of vitamin C, $400 \mathrm{IU}$ of vitamin $\mathrm{E}$ and $15 \mathrm{mg}$ of $\beta$-carotene), group 2 - zinc ( $80 \mathrm{mg}$ in the form of zinc oxide) and copper ( $2 \mathrm{mg}$ in the form of copper oxide), group 3 - antioxidants plus zinc, and group 4 - placebo. Subjects with extensive intermediate drusen, large drusen or non-central geographic atrophy in one or both eyes, or with advanced AMD, or with visual acuity $<20 / 32$ in one eye, treated with anti-oxidants in combination with zinc, had the lowest risk of AMD progression or loss of visual acuity by 15 or more letters. The beneficial effect of antioxidants combined with zinc resulted in a $25 \%$ decrease in the relative risk of AMD progression to advanced stage. The reduction of the risk of AMD progression in the group receiving only antioxidants was $17 \%$, while for those receiving only zinc $-21 \%$. The $\beta$-carotene dose used in this study was $15 \mathrm{mg} / 24 \mathrm{~h}$. Other studies using similar $\beta$-carotene doses in high risk lung cancer groups (smokers) have shown increased cancer incidence and mortality [31]. The study safety monitoring committee advised smokers to stop taking $\beta$-carotene-containing medicines.

The following study AREDS2, published in 2013, included about $70 \%$ of the participants previously randomised in the AREDS study. $\beta$-carotene was removed from the original AREDS formula due to its potential carcinogenic effect in smokers, and the dose of zinc was also reduced to 25 mg. AREDS2 formula was enriched with omega-3 acids (DHA, EPA), lutein and zeaxanthin. The mean study duration was 5 years. The AREDS2 study, similarly to AREDS, confirmed the efficacy in reducing the risk of AMD progression in patients who supplemented their diet with a set of antioxidants, such as vitamin $C$, vitamin $\mathrm{E}$, zinc, copper, with the addition of lutein $(10 \mathrm{mg})$, zeaxanthin (2 $\mathrm{mg}$ ), and omega-3 acids (DHA, EPA) versus the original AREDS formula [21].

\section{CONCLUSIONS}

The research presented in this paper indicates an important role of diet supplementation in AMD.

In conclusion, in patients with AMD it is worth recommending supplementation with ingredients such as: lutein, zeaxanthin, omega-3 acids, zinc, folic acid and vitamins D, $\mathrm{B}_{6}, \mathrm{~B}_{12}, \mathrm{C}, \mathrm{E}$, as well as a healthy lifestyle and limited eye exposure to UV radiation. The vitamins, minerals and carotenoids discussed in this paper have anti-inflammatory and anti-oxidative properties, and therefore they reduce the risk of AMD progression. In the elderly, due to poor absorption, achieving optimal levels of vitamins, minerals and carotenoids can be challenging. For these patients, the alternative is supplementation of these ingredients. It is therefore appropriate to ensure an adequate level of these substances in the diet of AMD patients. 


\author{
CORRESPONDENCE \\ Iwona Kusz vel Sobczuk, MD \\ Medical University of Lublin, \\ Department of Retinal and Vitreous Surgery \\ 20-079 Lublin, Chmielna 1 \\ e-mail: iwona.kuszvelsobczuk@wp.pl
}

\section{ORCID}

Iwona Kusz vel Sobczuk - ID - https://orcid.org/0000-0002-7047-4696 Anna Święch - ID - https://orcid.org/0000-0002-2238-6966

\section{References}

1. Kauppinen A, Paterno JJ, Blasiak J et al. Inflammation and its role in age-related macular degeneration. Cell Mol Life Sci. 2016; 73: 1765-86.

2. Klein R, Klein BE, Tomany SC et al. Ten-year incidence of age-related maculopathy and smoking and drinking: The Beaver Dam Eye Study. Am J Epidemiol. 2002; 156: 589-98.

3. Alsalem JA, Patel D, Susarla R et al. Characterization of Vitamin D Production by Human Ocular Barrier Cells. Invest Ophthalmol Vis Sci. 2014; 55(4): 2140-7.

4. Hewison M. An update on vitamin D and human immunity. Clin Endocrinol (Oxf). 2012; 76: 315-25.

5. Jeffery LE, Burke F, Mura M et al. 1,25-Dihydroxyvitamin D3 and IL-2 combine to inhibit T cell production of inflammatory cytokines and promote development of regulatory T cells expressing CTLA-4 and FoxP3. J Immunol. 2009; 183: 5458-67.

6. Bartels $L E$, Hvas $C L$, Agnholt J et al. Human dendritic cell antigen presentation and chemotaxis are inhibited by intrinsic 25-hydroxy vitamin D activation. Int Immunopharmacol. 2010; 10: 922-8.

7. Kaarniranta K, Pawlowska E, Szczepanska J et al. Can vitamin D protect against age-related macular degeneration or slow its progression? Acta Biochim Pol. 2019; 66(2): 147-58.

8. Millen AE, Meyers KJ, Liu Z et al. Association between vitamin D status and age-related macular degeneration by genetic risk. JAMA Ophthalmol. 2015; 133(10): 1171-9.

9. Rusińska A, Płudowski P, Walczak M et al. Vitamin D Supplementation Guidelines for General Population and Groups at Risk of vitamin D Deficiency in Poland - Recommendations of the Polish Society of Pediatric endocrinology and Diabetes and the expert Panel with Participation of National Specialist Consultants and Representatives of Scientific Societies - 2018 Update. Front Endocrinol (Lausanne). 2018; 9: 246.

10. Li LH, Chung-Yung Lee J, Hang Leung H et al. Lutein Supplementation for Eye Diseases. Nutrients. 2020; 12(6): 1721.

11. Renzi LM, Hammond BR Jr, Dengler M et al. The relation between serum lipids and lutein and zeaxanthin in the serum and retina: Results from cross-sectional, case-control and case study designs. Lipids Health Dis. 2012; 11: 33.

12. Li B, George EW, Rognon GT et al. Imaging lutein and zeaxanthin in the human retina with confocal resonance Raman microscopy. Proc Natl Acad Sci USA. 2020; 117: 12352-8.

13. Li SY, Fu ZJ, Lo AC. Hypoxia-induced oxidative stress in ischemic retinopathy. Oxid Med Cell Longev. 2012; 2012: 426769.

14. Junghans A, Sies H, Stahl W. Macular pigments lutein and zeaxanthin as blue light filters studied in liposomes. Arch Biochem Biophys. 2001; 391: 160-4.

15. Krinsky NI, Landrum JT, Bone RA. Biologic mechanisms of the protective role of lutein and zeaxanthin in the eye. Ann Rev Nutr. 2003; 23: 171-201.

16. Kijlstra A, Tian Y, Kelly ER et al. Lutein: More than just a filter for blue light. Prog Retin Eye Res. 2012; 31: 303-15.

17. Tian Y, Kijlstra A, Webers CAB et al. Lutein and Factor D: Two intriguing players in the field of age-related macular degeneration. Arch Biochem Biophys. 2015; 572: 49-53.

18. Bian Q, Gao S, Zhou J et al. Lutein and zeaxanthin supplementation reduces photooxidative damage and modulates the expression of inflammation-related genes in retinal pigment epithelial cells. Free Radic Biol Med. 2012; 53: 1298-307.

19. Fung FK, Law BY, Lo AC. Lutein Attenuates Both Apoptosis and Autophagy upon Cobalt (II) Chloride-Induced Hypoxia in Rat Muller Cells. PLoS ONE. 2016; 11: e0167828.

20. Gale CR, Hall NF, Phillips DIW et al. Lutein and Zeaxanthin Status and Risk of Age-Related Macular Degeneration. Invest Ophthalmol Vis Sci. 2003; 44: 2461-5.

21. Age-Related Eye Disease Study 2 Research Group. Lutein + zeaxanthin and omega-3 fatty acids for age-related macular degeneration: the age-related eye disease study 2 (AREDS2) randomized clinical trial. JAMA. 2013; 309: 2005-15. 
22. Seddon JM, George S, Rosner B. Cigarette Smoking, Fish Consumption, Omega-3 Fatty Acid Intake, and Associations With Age-Related Macular Degeneration The US Twin Study of Age-Related Macular Degeneration. Arch Ophthalmol. 2006; 124(7): 995-1001.

23. Seddon JM, Rosner B, Sperduto RD et al. Dietary fat and risk for advanced age-related macular degeneration. Arch Ophthalmol. 2001; 119: 1191-9.

24. Austin RC, Lentz SR, Werstuck GH. Role of hyperhomocysteinemia in endothelial dysfunction and atherothrombotic disease. Cell Death Differ. 2004; 11(suppl 1): S56-64.

25. Christen WG, Glynn RJ, Chew EY et al. Folic acid, pyridoxine, and cyanocobalamin combination treatment and age-related macular degeneration in women: the Women's Antioxidant and Folic Acid Cardiovascular Study. Arch Intern Med. 2009; 169: 335-41.

26. Gopinath B, Flood VM, Rochtchina E et al. Homocysteine, folate, vitamin B-12, and 10-y incidence of age-related macular degeneration. Am J Clin Nutr. 2013; 98(1): 129-35.

27. Christen WG, Glynn RJ, Chew EY et al. Folic Acid, Vitamin B6, and Vitamin B12 in Combination and Agerelated Macular Degeneration in a Randomized Trial of Women. Arch Intern Med. 2009; 169(4): 335-41.

28. Herbert V, Bigaouette J. Call for endorsement of a petition to the Food and Drug Administration to always add vitamin B-12 to any folate fortification or supplement. Am J Clin Nutr. 1997; 65: 572-3.

29. Smailhodzic D, Van Asten F, Blom A et al. Zinc supplementation inhibits complement activation in age-related macular degeneration. PLoS One. 2014; 9(11): e112682.

30. Dharamdasani Detaram H, Mitchell P, Russell J et al. Dietary zinc intake is associated with macular fluid in neovascular age-related macular degeneration. Clin Exp Ophthalmol. 2020; 48(1): 61-8.

31. Age-Related Eye Disease Study Research Group. A Randomized, Placebo-Controlled, Clinical Trial of High-Dose Supplementation With Vitamins C and E, Beta Carotene, and Zinc for Age-Related Macular Degeneration and Vision Loss: AREDS Report No. 8. Arch Ophthalmol. 2001; 119(10): 1417-36.

\section{Authors' contributions:}

Iwona Kusz vel Sobczuk: conceptualization, writing and preparation of an original project,

review and editing; Anna Święch: review and editing.

Conflict of interest:

None.

Financial support:

None.

Ethics:

The content presented in the article complies with the principles of the Helsinki

Declaration, EU directives and harmonized requirements for biomedical journals. 